\title{
Prognostic Roles of C-Reactive Protein and Erythrocyte Sedimentation Rate As Acute Phase Reactants in Mentally Challenged Subjects
}

\section{Adedeji David Atere ${ }^{1,3}$, Bashiru S. A. Oseni², Ifelola Patience Adebua ${ }^{3}$, Joshua Seun Fapohunda1, and Idomeh Festus Aigbokheo ${ }^{4}$}

${ }^{1}$ Department of Medical Laboratory Science, University of Benin, Benin City, Edo State, Nigeria ${ }^{2}$ Department of Biomedical Sciences, Ladoke Akintola University of Technology, Osogbo, Osun State

${ }^{3}$ Department of Medical Laboratory Science, Achievers University, Owo, Ondo State, Nigeria

${ }^{4}$ University of Benin Teaching Hospital, Benin City, Edo State, Nigeria

\section{Abstract}

Background: A mental disorder is a psychiatric disease that presents as mild or severe disturbances in a person's behavior, mood, or thought. Mental illnesses are very common because of excessive stress. Recent studies show that mental illnesses are on the rise generally because of increasing stress. In Nigeria, medical records suggest an upsurge in mental health cases since the onset of the country's economic

Corresponding Author: Adedeji David Atere; email: ateread@gmail.com

Received 12 November 2018 Accepted 17 December 2018 Published 26 December 2018

Production and Hosting by Knowledge E

(c) Adedeji David Atere et al. This article is distributed under the terms of the Creative Commons Attribution License, which permits unrestricted use and redistribution provided that the original author and source are credited.

Editor-in-Chief:

Prof. Mohammad A. M. Ibnouf downturn and the consequent trauma following it. The erythrocyte sedimentation rate (ESR) is an indirect marker of serum acute-phase protein concentrations, whereas C-reactive protein (CRP) is a direct protein measurement and it is inherently more well-defined. This study thus evaluated the roles of ESR and CRP as sensitive markers of inflammation and correlated their levels with severity stratification and prognosis in schizophrenic patients.

Materials and Methods: This is a case-control study, and it was carried out on 40 patients diagnosed with schizophrenia. Thirty (30) controls were matched for age and sex; 8 millimeters of venous blood was collected from each participant using ante-cubital veins. Samples collected were dispensed into appropriate bottles for analysis of CRP and hematological parameters using standard laboratory procedures. Statistical analysis of the data was done appropriately and $P$-value of less than 0.05 was considered as significant.

Results: At the end of the study, the mean granulocytes, ESR, and CRP were significantly higher in schizophrenia cases than in the controls, while the mean PCV, RBC, and hemoglobin were significantly lower in the subjects. CRP had a higher area than ESR under the ROC curve (AUROC).

Conclusion: This study thus evaluated the role of ESR and CRP as sensitive markers of inflammation and acute phase reactants in schizophrenic patients. The findings showed that CRP was a better diagnostic or predictor treatment outcome of schizophrenia. 
Keywords: schizophrenia, acute phase reactants, C-reactive protein, erythrocyte sedimentation rate, inflammation

\section{Introduction}

Schizophrenia is a mental disorder that is characterized by a breakdown of thought processes and by poor emotional responsiveness, with mild or severe disturbances in a person's behavior, mood, or thought [1]. Even though schizophrenia has long been described as a disease of 'unknown etiology' [2], it had been documented to be associated with multiple genetic and environmental risk factors, including drug and alcohol abuse, prenatal infections, and malnutrition [1, 3]. Generally, a mental disorder appears in late adolescence or early adulthood. However, it can emerge at any time in life. Its common features include auditory hallucinations, paranoid or bizarre delusions, or disorganized speech and thinking, accompanied by significant social or occupational dysfunction $[1,4]$. Most people feel that mental disorders are rare and would not happen to them or the members of their family. This is largely incorrect! Mental illnesses are very common. About 30\% of Nigerians suffer from or are liable to different mental disorders, but the majority of them are undiagnosed [5]. Nigeria with a population of nearly 170 million has about 1.7 million people suffering from schizophrenia [6]. This figure reached before 2014 has risen to a new level following the economic slump and the accompanying stress the country has suffered since 2016 which can lead to a state of depression. Depression is a state of low mood and aversion to activity that affects thoughts, behavior, and physical well-being [7]. It remains a health issue of public importance. It affects about 121 million people and is recognized as the leading cause of disability worldwide [8]. Absolute poverty, limited public health services, civil unrest, gender inequality, and multiple medical problems are some of the identified causes of depression [9].

Despite decades of intensive research, diagnosis, and identification of patients with severe form of depression, it still remains a challenge due to the lack of routinely effective laboratory tests. This is further compounded by the unfavorable prognosis of depression as relapse/recurrence rate could be as high as $87 \%$ over 15 years with at least $40 \%$ not responding to initial treatment [10]. The search for serumbased biological marker for the diagnosis of depression or schizophrenia has been on since 1960 when urinary excretion of metabolites of catecholamines, particularly 
norepinephrine, was used to classify major mood disorders [11]. Laboratory investigations have linked mental problems with excessive stress. High cortisol levels reflect a higher degree of stress, but are dependent on the integrity of the HPA-axis [12]. Also, elevated plasma levels of arginine vasopressin (AVP) which is another stress hormone produced through HPA axis activity has been reported in schizophrenic patients [13]. Copeptin is a sensitive and stable surrogate marker for AVP release. It reflects the individual stress level that plays a major role in depression and schizophrenia $[5,14]$.

Acute phase proteins (APP) are blood proteins primarily synthesized by hepatocytes as part of the acute phase response (APR). The APR is a part of the early defense or innate immune system, which is triggered by different stimuli including trauma, infection, stress, neoplasia, and inflammation. The APR results in a complex systemic reaction with the goal of re-establishing homeostasis and promoting healing [15]. CRP has been inherently well-defined as a direct marker of acute protein measurement, while erythrocyte sedimentation rate (ESR) is an indirect biomarker of serum acutephase protein concentrations. CRP is an acute phase reactant. It is a protein made by the liver and released into the bloodstream within $24-48$ hours after tissue injury, at the start of an infection, or any inflammatory condition [16]. The level of CRP can rise a thousand-fold in response to an inflammatory condition, and its rise in the blood can precede pain, stress, or other clinical indicators [17]. CRP is not diagnostic of any condition, but it can be used together with symptoms and other tests to evaluate an individual for an acute or chronic inflammatory condition [18, 19]. CRP is a 'marker' for systemic inflammation [20], and thus would be useful as a prognostic marker in management of schizophrenic subjects. ESR is a laboratory test that measures erythrocytes distance in a vertical tube (in millimeters) under the influence of gravity force in one hour. It is a simple and an inexpensive test for an indirect measurement of serum acute-phase protein concentrations that responds slowly to inflammatory stimuli. The ESR depends on the aggregation of red blood cells (RBCs) and rouleaux formation [21]. As a patient's condition worsens or improves, the ESR responds slowly, because fibrinogen, the major contributor to the short-term increase in ESR, has a halflife of days (depending on other hemostatic factors), and immunoglobulins (strongly contributing to the elevation of ESR in chronic inflammatory states) have half-lives of weeks under normal physiologic states. This process can result in a significant lag between clinical changes and ESR values [21]. This study will thus evaluate the roles of ESR and CRP as both sensitive markers of inflammation and correlate their levels with severity stratification and prognosis in mentally challenged patients. 


\section{Materials and Methods}

\subsection{Study design}

This is a case control study.

\subsection{Subjects}

The ethical approval with registration number ERC/2018/04/04/116B from the Federal Teaching Hospital, Ido-Ekiti (FTHI) Ethics and Research Committee was duly obtained for this study and also the informed consent or otherwise assent was taken from each participant or relative, respectively. Thereafter, forty (40) schizophrenic subjects and thirty (30) age- and sex-matched controls were randomly recruited into the study. All the participants were between the ages of 18 and 70 years. The schizophrenic patients were recruited from the Psychiatry Clinic of the FTHI, while the control group comprised the members of the staff of the Hospital who had no symptoms of schizophrenia. The schizophrenia group was diagnosed according to the Diagnostic and Statistical Manual of Mental Disorders, fourth edition, text revision (DSM-4TR) by clinically trained and experienced psychiatrists. A trained psychiatrist administered the Positive and Negative Syndrome Scale (PANSS) and recorded scores appropriately. PANSS is a medical scale used for measuring symptom severity of patients with schizophrenia [22].

\subsection{Inclusion criteria (cases and controls)}

The criteria for the inclusion of cases were participants that gave informed consent, satisfied PANSS for the diagnosis of schizophrenia, subjects whose positive and negative scales were $\leq 7 x \geq 49$ (Schizophrenia) and aged 18 to 70 years. The criteria for the inclusion of controls were participants aged 18 to 70 years and whose PANSS scale score was less than 7 (Schizophrenia).

\subsection{Exclusion criteria (cases and controls)}

The criteria for the exclusion of cases and controls were geriatric subjects (> 70 years) and subjects less than 18 years, subjects with concurrent use of non-steroidal antiinflammatory agents, steroids, antidepressants, antipsychotic medications, and anticonvulsants 3 weeks before presentation, subjects with previous history of mania or 
hypomania, or any concurrent psychotic symptoms, subjects with pregnancy, presence of serious and/or unstable medical disorders, previous history of Addison's disease, Cushing's disease or thyroid diseases or diabetic and hypertensive subjects.

\subsection{Collection/sampling technique and storage of sample}

Eight milliliters $(8 \mathrm{ml})$ of venous blood was collected from each subject using aseptic procedure. Two ( $2 \mathrm{ml}$ ) of venous blood was dispensed into a sterile ESR bottle containing 3.2\% tri-sodium citrate solution in a ratio of blood-citrate, 5:1 (v/v) as an anticoagulant and gently mixed by inverting the container several times for the determination of ESR; $3 \mathrm{ml}$ of the samples collected was dispensed into plain bottles and allowed to retract. Each sample was spun at $4000 \mathrm{rpm}$ for 5 minutes to obtain serum that was stored at $-20 \mathrm{C}$ until analysis. Similarly, three milliliters $(3 \mathrm{ml}$ ) of venous blood left over was dispensed into ethylene diamine acetate bottle for hematological parameters analysis.

\subsection{Analytical methods and procedures}

The serum level of CRP was determined using enzyme immunosorbent assay (ELISA) as described by Johnson et al. [23], and standard methods were employed to determine ESR and full blood count (FBC), using Westergren method and hematological analyzer, respectively.

\subsection{Statistical analysis}

A statistical package for social scientist (SPSS) 23.0 version was used for the appropriate analysis of the data. The distribution of the variables was assessed using histogram with standard curve. Variables with Gaussian distribution were compared between groups using Student's $t$-test. However, to adjust for confounding factors, Analysis of variance (ANOVA) was used to compare differences between the schizophrenic groups based on the year of diagnosis. A graphical plot of sensitivity (Receiver Operating Curve; ROC) was done and an area under ROC (AUROC) of each marker (CRP and ESR) was compared using pair-wise comparison. Spearman correlation was used to test the association between inflammatory markers (CRP and ESR) and other variables in exposed groups. $P$-values less than 0.05 was considered significant. 


\section{Results}

A total number of 70 subjects were studied. They comprised 40 schizophrenic subjects with mean age of $40.65 \pm 12.32$ years and 30 controls with mean age of $38.33 \pm$ 11.93 years. Figure 1 shows the age and sex distribution of the recruited population in percentage (\%). There were 16 females and 24 males in the schizophrenic group, and 13 females and 17 males in the non-schizophrenic groups. Thus, females constituted $41.42 \%$, while males constituted $58.58 \%$ overall. Comparing the mean hematological parameters and CRP in schizophrenic subjects and controls using independent student $T$-test, the mean GRA, ESR, and CRP were significantly higher among schizophrenia, while the mean PCV, RBC, and HBG were significantly lower (Table 1). The schizophrenic subjects were further classified into five groups based on the years of diagnosis. It was observed, as shown in Table 2, that there were no significant differences in the mean of hematological indices and CRP within the three groups, using One Way Analysis of Variance (ANOVA).

Table 3 shows spearman correlation between hematological parameters with ESR and CRP schizophrenic subjects. CRP showed positive correlation with GRA and negative correlation with LYMP, while ESR only had inverse correlation with PCV, RBC, and HGB. The diagnostic performance of ESR and CRP were determined. CRP had higher Area under the ROC curve (AUROC) than ESR as shown in Figure 2.

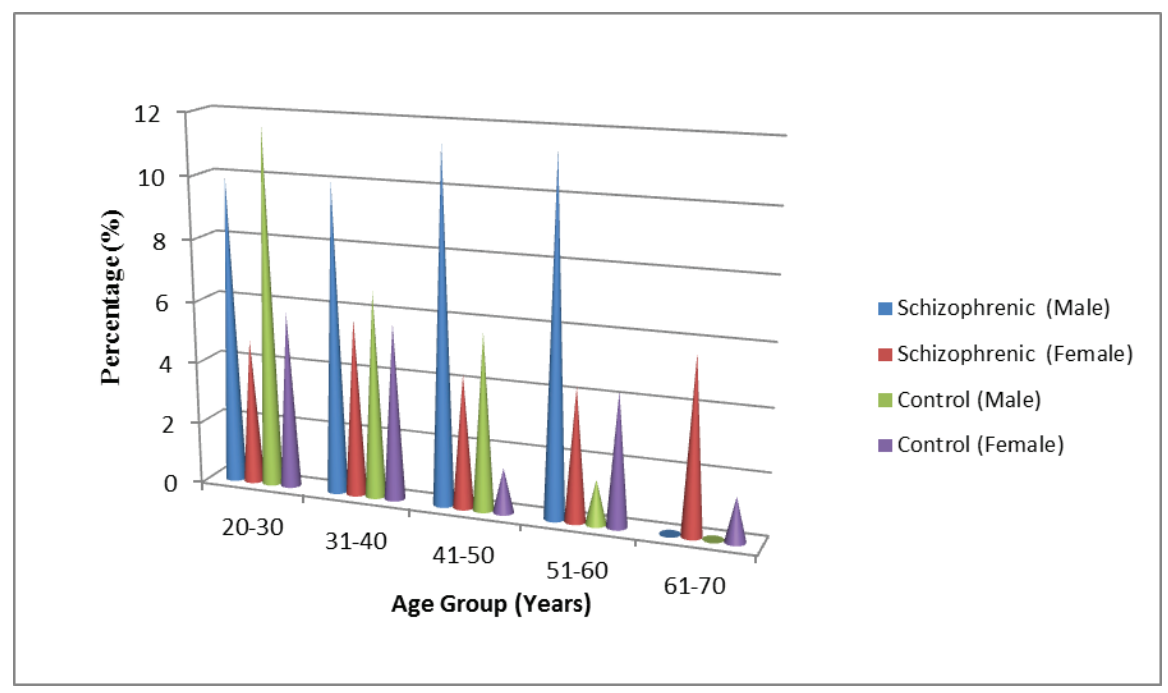

Figure 1: Age and Sex distribution of the recruited population in percentage (\%).

\section{Discussion and Conclusion}


TABLE 1: Comparison of mean age, hematological parameters, and CRP among schizophrenic and nonschizophrenic subjects.

\begin{tabular}{|c|c|c|c|c|}
\hline Parameters & $\begin{array}{l}\text { Schizophrenic } \\
\text { subjects }(n=40)\end{array}$ & $\begin{array}{l}\text { Non-schizophrenic } \\
\text { subjects }(n=30)\end{array}$ & P-value & Rmk \\
\hline Age (Years) & $40.65 \pm 12.32$ & $38.33 \pm 11.93$ & 0.433 & NS \\
\hline $\operatorname{PCV}(\%)$ & $38.34 \pm 4.09$ & $40.89 \pm 2.85$ & 0.005 & $\mathrm{~S}$ \\
\hline $\operatorname{RBC}\left(X 10^{12} / L\right)$ & $4.38 \pm 0.50$ & $4.76 \pm 0.49$ & 0.003 & $\mathrm{~S}$ \\
\hline HBG (g/dl) & $12.83 \pm 1.38$ & $13.49 \pm 1.17$ & 0.040 & $\mathrm{~S}$ \\
\hline MCV (fl) & $88.28 \pm 6.00$ & $85.80 \pm 4.87$ & 0.069 & NS \\
\hline MCH (pg) & $29.18 \pm 2.75$ & $28.46 \pm 2.20$ & 0.243 & NS \\
\hline MCHC (g/dl) & $32.93 \pm 1.21$ & $33.00 \pm 0.98$ & 0.792 & NS \\
\hline 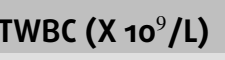 & $6.30 \pm 1.91$ & $6.07 \pm 1.57$ & 0.591 & NS \\
\hline LYMP (\%) & $40.37 \pm 10.22$ & $44.05 \pm 10.13$ & 0.139 & NS \\
\hline GRA (\%) & $48.32 \pm 9.53$ & $43.91 \pm 8.22$ & 0.046 & $S$ \\
\hline PLT (X 10 1 /L) & $178.53 \pm 59.06$ & $205.30 \pm 61.45$ & 0.069 & NS \\
\hline ESR (mm/Hr) & $37.73 \pm 23.33$ & $12.20 \pm 9.06$ & 0.000 & $\mathrm{~S}$ \\
\hline CRP ( $1 \mathrm{~g} / \mathrm{ml})$ & $1.27 \pm 0.32$ & $1.07 \pm 0.12$ & 0.002 & $S$ \\
\hline \multicolumn{5}{|c|}{ Note: * Significant at $P \leq 0.05$} \\
\hline \multicolumn{5}{|c|}{$\begin{array}{l}\text { PCV = Packed cell volume; } \mathrm{RBC}=\text { Red blood cell; } \mathrm{HGB}=\text { Hemoglobin concentration; } M C V \\
\text { Mean cell volume; } M C H=\text { Mean cell haemoglobin; } M C H C=\text { Mean cell hemoglobin concentration } \\
\text { TWBC = Total white blood cell; LYMP = Lymphocyte; GRA = Granulocytes; PLT = Platelets; ESR } \\
\text { Erythrocytes sedimentation rate; } C R P=\text {-reactive protein; Rmk = Remark; } S=\text { Significant; an } \\
\text { NS = Not significant. }\end{array}$} \\
\hline
\end{tabular}

TABLE 2: Comparison of mean hematological indices and CRP based on years of diagnosis among the schizophrenic subjects.

\begin{tabular}{|c|c|c|c|c|c|c|}
\hline $\begin{array}{l}\text { Years of } \\
\text { diagnosis } \\
\text { Parameters }\end{array}$ & $0-5(n=20)$ & $6-10(n=7)$ & $11-15(n=4)$ & $16-20(n=6)$ & $>20(n=3)$ & p-value \\
\hline Age (Years) & $38.75 \pm 12.20$ & $38.71 \pm 14.44$ & $39.25 \pm 14.32$ & $47.17 \pm 3.66$ & $46.67 \pm 18.61$ & 0.562 \\
\hline PCV (\%) & $38.58 \pm 4.47$ & $37.40 \pm 4.45$ & $37.28 \pm 2.94$ & $39.57 \pm 2.58$ & $37.90 \pm 6.00$ & 0.877 \\
\hline $\operatorname{RBC}\left(X \mathbf{1 0}^{12} / \mathrm{L}\right)$ & $4.42 \pm 0.56$ & $4.30 \pm 0.54$ & $4.31 \pm 0.29$ & $4.36 \pm 0.45$ & $4.48 \pm 0.64$ & 0.978 \\
\hline HBG (g/dl) & $13.00 \pm 1.54$ & $12.34 \pm 1.71$ & $12.58 \pm 0.95$ & $13.12 \pm 0.99$ & $12.63 \pm 0.74$ & 0.824 \\
\hline MCV (fl) & $88.90 \pm 5.85$ & $86.71 \pm 9.09$ & $86.25 \pm 4.57$ & $90.17 \pm 4.07$ & $86.67 \pm 4.73$ & 0.763 \\
\hline MCH (pg) & $29.68 \pm 2.61$ & $28.64 \pm 3.93$ & $28.20 \pm 2.11$ & $29.63 \pm 1.55$ & $27.50 \pm 3.55$ & 0.625 \\
\hline MCHC (g/dl) & $33.19 \pm 1.16$ & $32.90 \pm 1.28$ & $32.83 \pm 0.97$ & $32.73 \pm 0.84$ & $31.77 \pm 2.21$ & 0.443 \\
\hline $\operatorname{TWBC}\left(X_{10}{ }^{9} / L\right)$ & $6.24 \pm 1.78$ & $6.71 \pm 2.47$ & $5.71 \pm 1.42$ & $6.55 \pm 2.35$ & $5.96 \pm 2.06$ & 0.929 \\
\hline LYMP (\%) & $39.50 \pm 10.96$ & $42.71 \pm 9.87$ & $33.93 \pm 10.13$ & $45.38 \pm 6.59$ & $39.23 \pm 12.41$ & 0.483 \\
\hline GRA (\%) & $48.19 \pm 10.78$ & $47.10 \pm 8.56$ & $54.33 \pm 6.06$ & $44.70 \pm 6.41$ & $51.20 \pm 12.65$ & 0.604 \\
\hline PLT (X 10 $\left.{ }^{9} / \mathrm{L}\right)$ & $181.45 \pm 65.17$ & $176.00 \pm 63.70$ & $182.50 \pm 80.69$ & $175.50 \pm 46.12$ & $165.67 \pm 2.52$ & 0.994 \\
\hline ESR (mm/Hr) & $32.50 \pm 22.96$ & $41.86 \pm 24.51$ & $34.50 \pm 23.24$ & $42.00 \pm 22.76$ & $58.67 \pm 23.69$ & 0.432 \\
\hline CRP $(\mu \mathrm{g} / \mathrm{ml})$ & $1.33 \pm 0.31$ & $1.25 \pm 0.44$ & $1.19 \pm 0.19$ & $1.18 \pm 0.23$ & $1.17 \pm 0.32$ & 0.865 \\
\hline
\end{tabular}


TABLE 3: Correlation between inflammatory markers, weight, body mass index, fasting blood sugar, and oxidative stress parameters in exposed groups.

\begin{tabular}{|c|c|c|}
\hline & ESR R-value $p$-value & CRP r-value p-value \\
\hline Age (Years) & 0.0710 .662 & -0.1860 .249 \\
\hline PCV (\%) & $-0.5660 .000^{*}$ & -0.0320 .845 \\
\hline $\operatorname{RBC}\left(X \mathbf{1 0}^{12} / \mathrm{L}\right)$ & $-0.5780 .000^{*}$ & 0.0490 .766 \\
\hline HBG (g/dl) & $-0.5230 .001^{\star}$ & 0.0430 .793 \\
\hline MCV (fl) & 0.1710 .292 & -0.0720 .660 \\
\hline MCH (pg) & 0.0640 .696 & -0.0290 .857 \\
\hline MCHC (g/dl) & -0.1260 .437 & 0.1380 .396 \\
\hline TWBC (X 109 /L) & 0.2340 .146 & -0.1840 .256 \\
\hline LYMP (\%) & -0.0490 .766 & $-0.2640 .027^{*}$ \\
\hline GRA (\%) & 0.1710 .292 & $0.2620 .029^{\star}$ \\
\hline PLT (X 10 $/ \mathrm{L})$ & -0.0460 .778 & -0.0440 .790 \\
\hline ESR $(\mathrm{mm} / \mathrm{Hr})$ & $1.000-$ & -0.2130 .186 \\
\hline
\end{tabular}

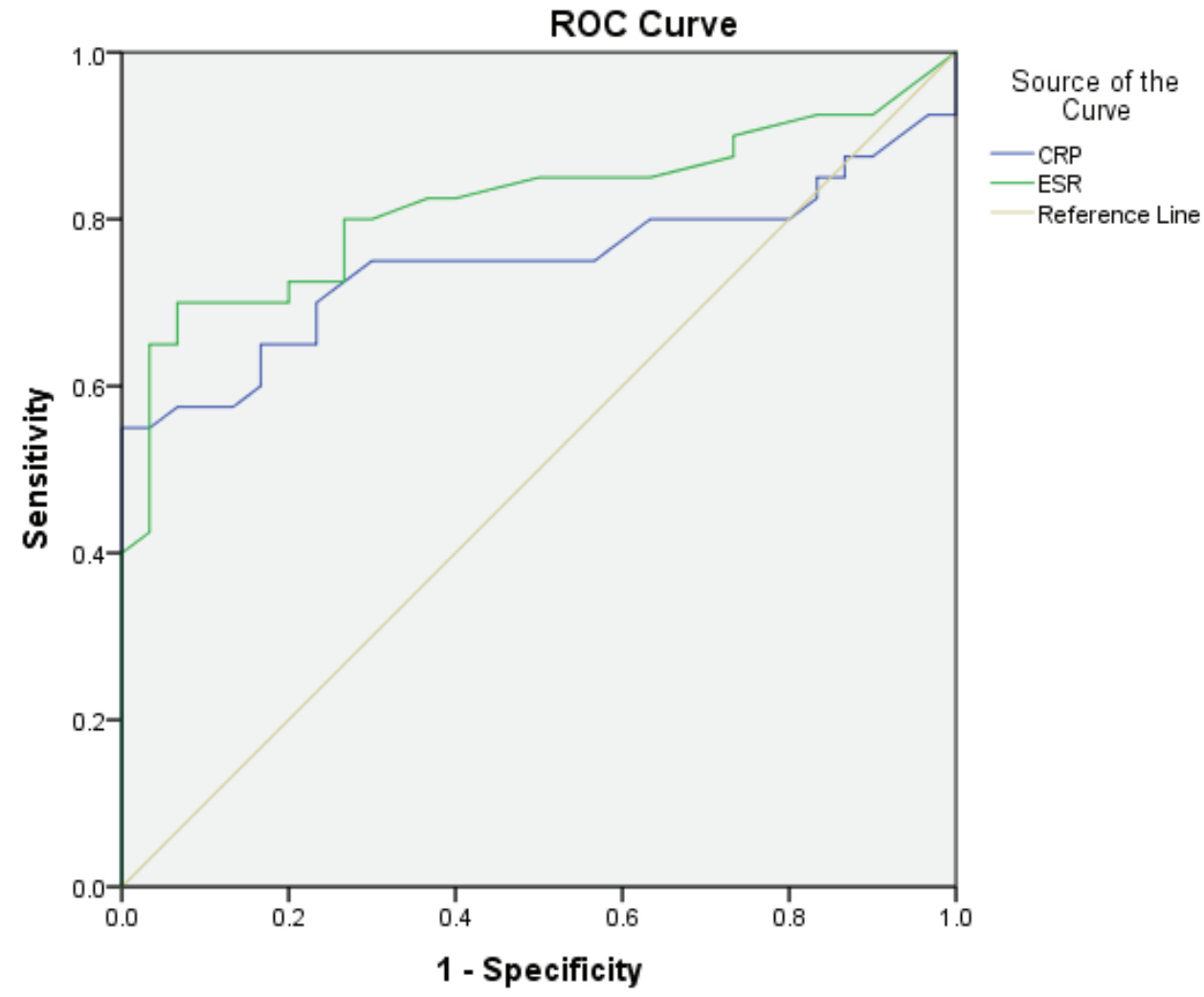

Diagonal segments are produced by ties.

Figure 2: The ROC curve of blood levels of CRP and ESR schizophrenic subjects. 


\subsection{Discussion}

Figure 1 shows the age and sex distribution of the recruited population in percentage $(\%)$. A total number of seventy (70) subjects consisted of forty (40) schizophrenic subjects with mean age of $40.65 \pm 12.32$ years and thirty $(30)$ control groups with mean age of $38.33 \pm 11.93$ years were studied. The subjects constituted of $41.42 \%$ and $58.58 \%$ females and males, respectively, overall; $31.42 \%$ of schizophrenic subjects in this study were youths within the age bracket of $18-40$ years. The major cause of mental challenge in Nigeria is traceable to either drug abuse, abject poverty, or any daily activities that could lead to depression [6]. Despite decades of intensive research, diagnosis, and identification of patients with severe form of mental disorders, it remains a challenge due to the lack of routinely useful laboratory test. There remains an urgent need to identify biomarkers that can determine the severity, aid in diagnosis, and probably predict the outcome of the treatment of these disorders.

The data in this study showed that the mean levels of granulocytes (GRA), ESR and C-reactive protein (CRP) were significantly higher in schizophrenic group in comparison with non-schizophrenic groups, while the mean PCV, RBC, and HBG were significantly lower among schizophrenic group. CRP is an acute phase reactant, produced by some inflammatory stimuli in the liver. It is mainly induced by interleukin 6 (IL-6), which is pro-inflammatory cytokines released into the bloodstream $[16,24]$. CRP is a 'marker' for systemic inflammation [20], and thus would be useful as a prognostic marker in the management of schizophrenic subjects. Increase in the mean levels of CRP in schizophrenia cases observed in this work is consistent with recent work where elevated levels of some inflammatory biomarkers, such as TNF- $\alpha$, was reported in schizophrenic patients $[25,26]$.

In the same vein, increase in mean levels of ESR observed among schizophrenia cases in comparison with control group confirms ESR roles as indirect marker of APP even though its response to inflammatory stimuli might be slow [27]. As a patient's condition worsens or improves, the ESR responds slowly, and thus elevated ESR has been reported to strongly contribute to chronic inflammatory states of the condition [21]. Melamed and Sirota [28] revealed that ESR increased in $17 \%$ of the patients with acute psychosis, which decreased to normal values after eight weeks of antipsychotic treatment in two-thirds of those patients, with the reduction of psychopathological manifestations. Here, using ESR with other markers, having a high ESR without a known physical illness might possibly serve as a biological indication of the remission and relapse of the disease. 
Results showed that CRP had a positive correlation with GRA, and ESR had a negative correlation with packed cell volume (PCV), red blood cells (RBC), and hemoglobin (HGB). Immune system dysfunctions and inflammatory processes show a link with the pathogenesis of schizophrenia as reported by Richard and Brahm [29]. Granulocytes are leukocytes of the innate immune system that have granules in their cytoplasm and they are neutrophil, basophil, and eosinophil. We could speculate that positive correlation between CRP and granulocytes are up-regulated as a compensatory mechanism to cope with the condition or response to treatment. This confirms the roles of CRP and granulocytes as acute protein reactant and immune system functions, respectively, in the management of mentally challenged subjects. On the contrary, ESR had negative correlation with PCV, RBC, and hemoglobin (HGB), and this corroborated the dependence of ESR on the aggregation of RBCs and rouleaux formation as documented by Calderon and Wener [21].

The diagnostic performance of ESR and CRP was determined using Area under the ROC curve (AUROC). CRP had higher Area under the ROC curve (AUROC) than ESR as shown in Figure 2. Even though ESR and CRP are both sensitive markers of inflammation and correlate with severity of inflammation, they are not specific to any particular illness [21]. The high levels of CRP in central nervous system have been reported to play an important role in some psychiatric dysfunctions, such as schizophrenia [30]. Similarly, Wang et al. [24] also reported that sensitivity analysis showed that serum and plasma CRP levels were increased moderately in studies involving only highsensitivity CRP assay. However, our finding, when we determined the diagnostic and prognostic performance of CRP and ESR using AUROC, showed that CRP, compared with $E S R$, had better performance. Thus, this finding is certainly of clinical importance since the measurement of CRP is widely available and affordable. Using CRP with symptoms and other laboratory tests to predict or monitor the treatment of schizophrenia would certainly improve the treatment condition of schizophrenic patients.

\subsection{Conclusion and recommendation}

This study thus evaluated the roles of ESR and CRP as sensitive markers of inflammation and acute phase reactants in schizophrenic patients. Also, CRP was found to be a better diagnostic or predictor treatment outcome of schizophrenia. This would definitely improve the treatment of psychiatric patients as well as prevent relapse and recurrence in the long term. 


\section{Acknowledgments}

The authors would like to thank all the participants, Resident Doctors, and staff of the Department of Psychiatry Clinic, the Federal Teaching Hospital, Ido-Ekiti (FTHI) for their support.

\section{Funding}

The research was self-sponsored.

\section{Conflict of Interest}

The authors declare that this manuscript was approved by all authors in its form and has no competing interests.

\section{References}

[1] Gonzalez-Liencres, C., Tas, C., Brown, E. C., et al. (2014). Oxidative stress in schizophrenia: A case-control study on the effects on social cognition and neurocognition. BMC Psychiatry, vol. 14, p. 268. Retrieved from http://www. biomedcentral.com/1471-244X/14/268

[2] Opler, M., Charap, J., Greig, A., et al. (2013). Environmental risk factors and schizophrenia. International Journal of Mental Health, vol. 42, no. 1, pp. 23-32. Retrieved from http://dx.doi.org/10.2753/IMHoo20-7411420102

[3] Brown, A. S. (2006). Prenatal infection as a risk factor for schizophrenia. Schizophrenia Bulletin, vol. 32, pp. 200-202.

[4] Van, O. S. and Kapur, S. (2009). Schizophrenia. Lancet, vol. 374, no. 9690, pp. 635645. DOI: 10.1016/S0140-6736(09)60995-8.

[5] Akinlade, K. S., Fagbomedo, F. O., and Rahamon, S. K. (2014). Serum copeptin and its diagnostic performance in schizophrenia. African Journal of Medical Sciences, vol. 43, pp. 259-264.

[6] Adegbaju, D. 1.7 million Nigerians have schizophrenia. Speech delivered by Dr. Dapo Adegbaju, a Consultant Psychiatrist at the Federal Neuro-Pschiatric Hospital, Yaba to mark the 2014 World Mental Health Day.

[7] Sandra, S. (1997). Depression: Questions You Hove - Answers You Need. People's Medical Society. 
[8] Abas, M. and Broadhead, J. (2001). Mental disorders in the developing world. British Medical Journal, vol. 308, p. 1052.

[9] Patel, V., Gwanzura, F., Simunyu, E., et al. (2001). The explanatory models and phenomenology of common mental disorder in Harare, Zimbabwe. Psychological Medicine, vol. 25, pp. 1191-1199.

[10] Beers, M. H., Fletscher, A. J., Jones, T. V., et al. (2005). Merck Manual. Houten: Bohn Stafleu van Loghum.

[11] Bahn, S., Guest, P., Harris, L., et al. Biomarkers for schizophrenia. Patent no: WO 2011121361A1. Filling date March 31, 2011.

[12] Akinlade, K. S., Atere, A. D., Olaniyi, J. A., et al. (2013). Serum copeptin and cortisol do not accurately predict sickle cell anaemia vaso-occlusive crisis as C-reactive protein. PLoS One, vol. 8, no. 11, e77913.

[13] Kawai, N., Baba, A., Suzuki, T., et al. (2001). Roles of arginine vasopressin and atrial natriuretic peptide in polydipsia-hyponatremia of schizophrenic patients. Psychiatry Research, vol. 101, no. 1, pp. 39-45.

[14] Katan, M., Nigro, N., Fluri, F., et al. (2011). Stress hormones predict cerebrovascular re-events after transient ischemic attacks. Neurology, vol. 76, no. 6, pp. 563-566.

[15] Ceron, J. J., Eckersall, P. D., and Martynez-Subiela, S. (2005). Acute phase proteins in dogs and cats: Current knowledge and future perspectives. Veterinary Clinical Pathology, vol. 34, pp. 85-99.

[16] Ridker, P. M., Danielson, E., and Fonseca, F. A. (2008). Rosuvastatin to prevent vascular events in men and women with elevated C-reactive protein. The New England Journal of Medicine, vol. 359, pp. 195-207.

[17] Schunkert, H. (2008). Elevated C-reactive protein in Atherosclerosis - Chicken or Egg? The New England Journal of Medicine, vol. 359, p. 18.

[18] Pearson, T. (2003). Markers of inflammation and cardiovascular disease. AHA/CDC Scientific Statement. Circulation, vol. 107, p. 499.

[19] Zacho, J., Hansen, A. T., Jensen, J. S., et al. (2008). Genetically elevated C-reactive protein and ischemic vascular disease. The New England Journal of Medicine, vol. 359, pp. 1897-1908.

[20] Stolz, D., Christ-Crain, M., Morgenthaler, N. G., et al. (2007). Copeptin, C-reactive protein, and procalcitonin as prognostic biomarkers in acute exacerbation of COPD. Chest, vol. 131, no. 4, pp. 1058-1067.

[21] Calderon, A. J. and Wener, M. H. (2012). Erythrocyte sedimentation rate and Creactive protein. Hospital Medicine Clinics, vol. 1, pp. 313-337. 
[22] Stefanović, V., Mihajlović, G., Nenadović, M., et al. (2015). The effect of antipsychotic drugs on nonspecific inflammation markers in the first episode of schizophrenia. Vojnosanitetski Pregled, vol. 72, no. 12, Pp. 1085-1092. DOI: 10.2298/VSP140526016S.

[23] Johnson, H. L., Chiou, C. C., and Cho, C. T. (1999). Applications of acute phase reactants in infectious diseases. Journal of Microbiology, Immunology and Infection, vol. 32, no. 2, pp. 73-82.

[24] Wang, Z., Li, P., Chi, D., et al. (2017). Association between C-reactive protein and risk of schizophrenia: An updated meta-analysis. Oncotorget, vol. 8, no. 43, pp. 7544575454 .

[25] Miller, B. J., Buckley, P., Seabolt, W., et al. (2011). Meta-analysis of cytokine alterations in schizophrenia: Clinical status and antipsychotic eects. Biological Psychiatry, vol. 70, pp. 663-671.

[26] Boll, K. M., Noto, C., Bonifácio, B. L., et al. (2017). Oxidative and nitrosative stress biomarkers in chronic schizophrenia. Psychiatry Research, vol. 253, pp. 43-48.

[27] Pavlovic, M., Babic, D., Rastovic, P., et al. (2013). Association of erythrocyte sedimentation rate and fibrinogen concentration with metabolic syndrome in a schizophrenic patients. Psychiatria Danubina, vol. 25, no. 1, pp. 51-55.

[28] Melamed, Y. and Sirota, P. (2000). Erythrocyte sedimentation rate in patients with schizophrenia. The Canadian Journal of Psychiatry, vol. 45, no. 10, p. 938.

[29] Richard, M. D. and Brahm, N. C. (2012). Schizophrenia and the immune system: Pathophysiology, prevention, and treatment. American Journal of Health-system Pharmacy, vol. 69, pp. 757-766.

[30] Najjar, S., Pearlman, D. M., Devinsky, O., et al. (2013). Neurovascular unit dysfunction with blood-brain barrier hyperpermeability contributes to major depressive disorder: A review of clinical and experimental evidence. Journal of Neuroinflammation, vol. 10, p. 142. 